\title{
Anastomosis primaria en enterocolitis necrotizante
}

\author{
MIGUEL GUELFAND CH. ${ }^{1}$, MARCELA SANTOS M. ${ }^{2}$, MARICARMEN OLIVOS P. ${ }^{3}$ \\ 1. Cirujano Pediátrico, Hospital Exequiel González Cortés - Clínica Las Condes. \\ 2. Cirujano Pediátrico, Hospital Exequiel González Cortés - Clínica Tabancura. \\ 3. Programa de Formación en Cirugía Pediátrica. Universidad de Chile. Hospital Exequiel González Cortés.
}

\begin{abstract}
Primary Anastomosis in Necrotizing Enterocolitis

Introduction: Necrotizing Enterocolitis (NE) is the most frequent gastrointestinal emergency among newborns (NB). Thirty percent of them require surgical treatment, with resection of the damaged intestinal segment and stoma formation. In some cases, primary resection and anastomosis can be considered. Objective: To review the use of primary anastomosis on NE in 2 pediatric centers, one public hospital and one private clinic. Patients and Method: A retrospective, descriptive study of all NB with NE managed with primary anastomosis at Hospital Exequiel González Cortés y Clínica Las Condes between december 2004 and december 2009. The population was divided into Group A: Unifocal, and Group B: Multifocal intestinal involvement. The following variables were evaluated and compared: gestational age, weight, use of peritoneal drains, characteristics of the resected segment, number of anastomoses, requirement of parenteral nutrition, postoperatory complications. Results: Sixty NB were surgically repaired with primary anastomosis. $12 \%$ presented birth weight $<1000$ grams, 22\% between 1000-1500 grams. In 18 patients 2 anastomoses were performed in different intestinal segments. Postoperatory complications included wound infection in 3 cases and dehiscence of the anastomosis in 1 case. $7 \%$ evolved with short loop syndrome. Mortality was 11,6\%, secondary to sepsis. Conclusions: In this experience, Primary Anastomosis in NE appears to be a safe option, with low morbimortality despite the age, weight, IP contamination or extension of the disease.

(Key words: Necrotizing enterocolitis, primary anastomosis, newborn).

Rev Chil Pediatr 2011; 82 (6): 520-524

\section{RESUMEN}

Introducción: Enterocolitis Necrotizante (ECN) es la emergencia gastrointestinal más común del recién nacido (RN), 30\% requiere tratamiento quirúrgico, con resección del segmento intestinal dañado, realizando luego una ostomía en la mayoría. En recientes casos la resección intestinal y anastomosis primeria han sido reportados en forma exitosa. Objetivo: Evaluar la experiencia del manejo con anastomosis primaria en ECN en 2 centros pediátricos, un Hospital público y una Clínica privada. Pacientes y Método: Estudio descriptivo retrospectivo. Se incluyó a RN del Hospital Exequiel González Cortés y Clínica Las Condes con ECN manejados con anastomosis primaria, entre diciembre de 2004 y diciembre de 2009. Se dividieron en Grupo A: Unifocal; Grupo B: Compromiso intestinal multifocal. Se comparó entre ambos grupos: edad gestacional,
\end{abstract}

Trabajo recibido el 19 de abril de 2011, devuelto para corregir el 30 de mayo de 2011, segunda versión el 20 de junio de 2011, tercera versión el 12 de agosto de 2011 aceptado para publicación el 02 de septiembre de 2011.

Correspondencia a:

Dra. Maricarmen Olivos Ch.

E-Mail: mguelfand@clc.cl 
peso, utilización de drenajes peritoneales, características del segmento resecado, número de anastomosis, requerimientos de nutrición parenteral, complicaciones postoperatorias. Resultados: Se sometió a cirugía con anastomosis primaria a 60 recien nacidos. El peso de nacimiento fue $<1000 \mathrm{~g}$ en el 12\%, $1000-1500 \mathrm{~g}$ el $22 \%$, y el resto $>1500$ g. En 18 pacientes del grupo B se realizaron 2 anastomosis en segmentos intestinales diferentes. Las complicaciones postoperatorias fueron infección de herida operatoria (n:3) y dehiscencia de anastomosis (n:1). Un 7\% evolucionó con síndrome de intestino corto. La mortalidad fue 11,6\%, en todos los casos secundaria a sepsis. Conclusiones: En esta experiencia la Anastomosis Primaria en ECN aparece como una opción segura, con baja morbimortalidad independiente de la edad, peso, contaminación intraperitoneal o extensión de la enfermedad.

(Palabras clave: Enterocolitis necrotizante, anastomosis primaria, recién nacidos).

Rev Chil Pediatr 2011; 82 (6): 520-524

\section{Introducción}

La Enterocolitis Necrotizante (ECN) es la emergencia gastrointestinal más común en el recién nacido, caracterizada por edema, ulceración y necrosis de la mucosa intestinal. En Recién Nacidos (RN), su incidencia fluctúa entre de 1 a $5 \%$ de los hospitalizados en Unidades de Cuidados Intensivos Neonatales (UCIN) siendo el 90 a 95\% de éstos, prematuros. La mortalidad varía de un 27 a 37\% en los pacientes que solo requieren tratamiento médico, alcanzado un $41-51 \%$ en los tratados quirúrgicamente ${ }^{1,2}$.

La etiopatogenia de este cuadro clínico no es aún del todo conocida. Su origen probablemente multifactorial considera la colonización intestinal fetal por patógenos, una respuesta linfocitaria exagerada y la inmadurez intestinal ${ }^{1,4}$.

En un $70 \%$ de los casos será suficiente el tratamiento médico para la resolución del cuadro, el cual consiste en reposo y descompresión intestinal por un periodo determinado, tratamiento antibiótico que cubra bacterias entéricas patógenas, corrección de trastornos metabólicos y hematológicos concurrentes, alimentación parenteral y control clínico médico y quirúrgico, además de control radiológico.

Alrededor de un $30 \%$ de los pacientes que presentan un cuadro de ECN requerirá de cirugía. El objetivo principal es la resección del segmento intestinal dañado, conservando la mayor cantidad de intestino vital ${ }^{3}$. La indicación quirúrgica aún es tema de controversia, pero la presencia de neumoperitoneo y el deterioro clínico del paciente se consideran una indicación absoluta de esta. Otras indicaciones de cirugía son: la presencia de masa abdominal y eritema de pared abdominal entre otros ${ }^{4,5}$.

No existe consenso en cuanto a la técnica quirúrgica más apropiada para el manejo de esta patología. Tradicionalmente en casi la totalidad de los casos se realiza una laparotomía para la resección del intestino comprometido asociado a una enterostomía proximal con o sin fístula mucosa .También está descrito en algunas publicaciones la utilización de anastomosis primaria posterior a la resección intestinal, sin dejar ostomías ${ }^{2,3,5}$ entre otras técnicas. En casos seleccionados en recién nacidos de muy bajo peso o de gran inestabilidad hemodinámica se utiliza la instalación de drenajes percutáneos en una primera etapa para la estabilización hemodinámica para luego realizar-eventualmente-una laparotomía ${ }^{7}$. En la literatura no existen estudios randomizados controlados (RTC) que comparen las distintas técnicas entre sí ${ }^{6}$.

Actualmente la técnica de elección es la anastomosis primaria, dado la favorable evolución observada en nuestros pacientes puesto que carece de las complicaciones asociadas a las enterotomías como la dermatitis periostomal, prolapso de ostomía, trastornos hidroelectrolíticos y el mal incremento ponderal, además de la necesidad de una segunda cirugía para la reconstitución del tránsito intestinal ${ }^{4}$. Dado que existen pocas publicaciones sobre la evolución y el seguimiento a largo plazo de pacientes manejados con anastomosis primaria decidimos presentar los resultados de esta experiencia.

El objetivo de este trabajo es analizar la ex- 
periencia en el manejo quirúrgico de pacientes con ECN en quienes se realizó resección intestinal con anastomosis primaria.

\section{Pacientes y Método}

Estudio descriptivo retrospectivo. Se analizaron las historias clínicas de RN del Hospital Exequiel González Cortés y Clínica Las Condes con diagnóstico de ECN que requirieron de laparotomía exploradora, con resección intestinal y sometidos a anastomosis primaria, en el período comprendido entre Diciembre de 2004 y Diciembre de 2009.

Se consideró como indicación de exploración la presencia de neumoperitoneo, eritema y/o edema de pared abdominal y mala respuesta al tratamiento médico. Ingresaron al estudio todos los $\mathrm{RN}$ con diagnóstico de enterocolitis necrotizante que cumplían con alguno de los criterios para exploración quirúrgica. Se excluyeron del análisis aquellos pacientes que en el intraoperatorio evolucionaron hemodinámicamente inestables, con enfermedad pan-intestinal y con vitalidad de los cabos con resecciones intestinales extensas dudosa o en que el tiempo quirúrgico se había prolongado. Se dividió a los pacientes en 2 grupos de acuerdo a la extensión de la enfermedad:

Grupo A: Unifocal (en donde la enfermedad se ubicaba en un solo segmento intestinal) y Grupo B: Multifocal (en donde la enfermedad se ubicaba en más de un segmento intestinal). En ambos grupos se evaluó las variables edad gestacional, peso, edad de inicio de cuadro, utilización de drenajes peritoneales, características del segmento resecado (longitud y ubicación anatómica), número de anastomosis realizadas, tiempo de requerimiento de nutrición parenteral y complicaciones post operatorias: recurrencia, infección de herida operatoria, dehiscencia de anastomosis, estenosis y síndrome de intestino corto.

Los resultados se entregan como promedio con su desviación estándar correspondiente cuando corresponde. Las comparaciones de promedios se hicieron con la prueba t de Student para muestras independientes.

\section{Resultados}

Durante el período analizado se realizó resección intestinal con anastomosis primaria a 60 pacientes con diagnóstico de ECN, lo que correspondió al 85\% del total de RN operados por ECN. De estos, 36 pacientes correspondieron al grupo A, y 24 al grupo B. El 15\% restante se manejó con ostomía, presentando este grupo la morbilidad asociada a la portación de una ostomía y la necesidad de una $2^{\circ}$ cirugía para reconstituir el tránsito intestinal.

La edad gestacional promedio fue de 31,2 semanas (rango 24 - 41 sem) y el peso de nacimiento promedio 1852 g (rango 550 - 3000 g), pesando el $12 \%$ menos de 1000 g, un $22 \%$ entre 1000 y $1500 \mathrm{~g}$, y el resto >1 $500 \mathrm{~g}$. La media de edad de presentación al inicio del cuadro fue de 5,3 días (2 a 18 días). La resección intestinal promedio fue de 19,6 cm (9 a $45 \mathrm{~cm}$ ), se resecó la válvula ileocecal en el $65 \%$ de los casos (n:39) y un $11 \%$ de los pacientes fue manejado con un drenaje peritoneal previo a la cirugía, todos con peso menor a 1500 gramos. En el grupo A se realizó una anastomosis en todos los casos, en el Grupo B el 75\% de los pacientes (n:18) requirió de dos anastomosis en segmentos intestinales diferentes.

En cuanto a la evolución postoperatoria se utilizó nutrición parenteral en todos los casos por 15,8 días en promedio (10-65). El 7\% (n:4) de los pacientes evolucionó con síndrome de intestino corto, todos correspondían al grupo multifocal. Se presentó dehiscencia del sitio de anastomosis en sólo 1 caso correspondiente al grupo B (multifocal) en un paciente al que se le habían realizado 2 anastomosis, ésta se resolvió quirúrgicamente sin complicaciones posteriores. Se observó una estenosis intestinal no relacionada con el sitio de anastomosis en un solo caso e infección de herida operatoria en 3 casos.

La estadía hospitalaria promedio fue de 38 días (rango 16 a 66 días). La mortalidad global fue de $11,6 \%$, en todos los casos secundaria a sepsis. La sobrevida para el Grupo A fue de 92\% y para el Grupo B 83\%.

En cuanto a la comparación de la evolución de los grupos con enfermedad unifocal y multifocal sólo se encontraron diferencias estadísticamente significativas en cuanto al número de 
anastomosis realizadas, 1 en grupo unifocal y 2 en el multifocal, y a la presencia de síndrome de intestino corto post quirúrgico, que sólo se observó en 4 pacientes del grupo multifocal. (p $<0,01)$. Las caracteristicas clínicas de los 2 grupos se detallan en la tabla 1.

En el seguimiento a largo plazo (1 a 6 años) se observaron en 3 casos obstrucción intestinal por adherencias que fueron resueltos exitosamente en forma quirúrgica.

\section{Discusión}

Frente a las alternativas de manejo de la Enterocolitis Necrotizante, nuestros resultados avalan la realización de la resección del o los segmentos intestinales no viables, con posterior anastomosis primaria como un procedimiento seguro, independiente de la edad, peso, contaminación intraperitoneal o extensión de la enfermedad del paciente. Los primeros resultados con esta técnica fueron publicados en 1979 por Kiesewetter, con una serie de nueve pacientes, de los cuales sobrevivieron $8^{11}$. Posteriormente se han reportado diferentes series con sobrevidas variables.

Por largo tiempo se consideró que en pacientes con diagnóstico de ECN, la resección de intestino inviable con la realización de una enterostomía proximal al sitio de resección era el procedimiento quirúrgico de elección. Se asumía que al realizar una anastomosis intestinal primaria, esta no sería vital en la presencia de septicemia y contaminación peritoneal franca, sumado a la incapacidad del cirujano en determinar exactamente la extensión de la enfermedad en la inspección del intestino durante la cirugía ${ }^{8}$. Se ha sugerido que el anastomosar intestino enfermo microscópicamente puede predisponer a fallas en la anastomosis y estenosis. Otras objeciones son la resección

Tabla 1. Características clínicas según grupo

\begin{tabular}{|c|c|c|c|}
\hline & $\begin{array}{c}\text { Grupo A: } \\
\text { Promedio (rango) } \\
\text { (n: 36) }\end{array}$ & $\begin{array}{c}\text { Grupo B: } \\
\text { Promedio (rango) } \\
\text { (n: 24) }\end{array}$ & $\mathbf{P}$ \\
\hline Edad Gestacional (semanas) & $\begin{array}{c}31 \pm 4,3 \\
(27-39)\end{array}$ & $\begin{array}{c}31,3 \pm 7,3 \\
(24-41)\end{array}$ & 0,45 \\
\hline $\begin{array}{l}\text { Peso }(\mathrm{g}) \\
>1500 \mathrm{~g} \\
1000-1500 \\
<1000\end{array}$ & $\begin{array}{c}1830 \pm 903(550-3000) \\
24 \\
8 \\
4\end{array}$ & $\begin{array}{c}1885 \pm 796(980-2900) \\
16 \\
5 \\
3\end{array}$ & 0,58 \\
\hline Edad de presentación (días) & $\begin{array}{c}5,5 \pm 4,3 \\
(2-18)\end{array}$ & $\begin{array}{c}5,1 \pm 2,9 \\
(3-12)\end{array}$ & 0,21 \\
\hline Resección intestinal (cm) & $\begin{array}{c}18 \pm 7,6 \\
(9-35)\end{array}$ & $\begin{array}{c}22 \pm 10,2 \\
(11-45)\end{array}$ & 0,32 \\
\hline Resección de Válvula Cecal & $58 \%$ & $75 \%$ & 0,19 \\
\hline Anastomosis única & 36 & 6 & 0,000001 \\
\hline Nutrición parenteral (días) & $\begin{array}{c}15 \pm 5,4 \\
(10-32)\end{array}$ & $\begin{array}{c}17 \pm 4,3 \\
(11-65)\end{array}$ & 0,24 \\
\hline Síndrome de intestino corto & 0 & 4 & 0,017 \\
\hline Recurrencia & 1 & 0 & 0,77 \\
\hline Estenosis & 1 & 0 & 0,77 \\
\hline Infección de herida operatoria & 1 & 2 & 0,77 \\
\hline Estadía hospitalaria & $\begin{array}{c}35 \pm 17,2 \\
(16-85)\end{array}$ & $\begin{array}{c}42 \pm 21,3 \\
(18-66)\end{array}$ & 0,32 \\
\hline Mortalidad & $8,3 \%$ & $16,6 \%$ & 0,33 \\
\hline
\end{tabular}

Grupo A: Unifocal; Grupo B: Multifocal. 
de más intestino del necesario, y el concepto de descompresión de la enterostomía, que se mantiene hasta ganar el peso óptimo antes de la reconstitución del tránsito.

Sin embargo se observan complicaciones de la enterostomía hasta en un 68\% de los pacientes estudiados ${ }^{9}$. Las complicaciones del ostoma incluyen excoriación de la piel circundante, prolapso, estenosis, necrosis, alteración del equilibrio hidroelectrolítico por altas pérdidas intestinales y falla en el crecimiento ${ }^{5}$.

A esto se agrega la necesidad de una segunda intervención para la reconstitución del tránsito. Esto ha llevado a plantear a la anastomosis primaria como una alternativa válida. Además, las estenosis intestinales, una complicación bien conocida de ECN, han sido reportadas con una menor incidencia después de la resección y anastomosis primaria. Se plantea como causa el rápido restablecimiento de la continuidad intestinal ${ }^{10}$.

En resumen, la anastomosis primaria en enterocolitis necrotizante surge como el tratamiento de elección en todos los casos que requieran de resección intestinal, ya sea con compromiso intestinal unifocal o multifocal, independiente de la localización y extensión del área necrótica o de la contaminación intraperitoneal, hecho que se demuestra en que solo en 1 caso presentó dehiscencia de anastomosis y en que el único caso de estenosis no fue en relación al sitio anastomótico. La realización de resección y anastomosis en dos segmentos intestinales no incide en la mortalidad. La utilización de esta técnica requiere de un cirujano con experiencia en casos de ECN y de las condiciones propias de cada paciente, no siendo una limitante el Peso de Nacimiento, puesto que un $33 \%$ de nuestra casuística fue menor a $1500 \mathrm{~g}$.

Esta serie apoya el concepto que la Anastomosis Primaria es una opción segura y con baja morbi-mortalidad, de acuerdo a lo que sugieren nuestros resultados, lo cual se traduce en una mejor calidad de vida para los pacientes. Los autores recomiendan así su realización como primera opción en pacientes con Ente- rocolitis Necrotizante enfrentados por equipos médicos con experiencia en esta patología.

La limitación del presente estudio radica en que se trata de un trabajo retrospectivo, $y$ no considera una evaluación comparativa de pacientes con similares características manejados con ambas técnicas quirúrgicas. Los excelentes resultados obtenidos en esta serie deben ser analizados a la luz de estas limitaciones.

\section{Referencias}

1. Srinivasan PS, Brandler MD, D'Souza A: Necrotizing enterocolitis. Clin Perinatol 2008; 35 (1): 251-72.

2. Blakely $M L$, Gupta H, Lally KP: Surgical management of necrotizing enterocolitis and isolated intestinal perforation in premature neonates. Semin Perinatol 2008; 32 (2): 122-6.

3. Guner YS, Chokshi N, Petrosyan M, Upperman JS, Ford $H R$, Grikscheit TC: Necrotizing enterocolitis--bench to bedside: novel and emerging strategies. Semin Pediatr Surg 2008; 17 (4): 255-65.

4. Singh $M$, Owen A, Gull S, Morabito A, Bianchi A: Surgery for intestinal perforation in preterm neonates: anastomosis vs stoma. J Pediatr Surg 2006; 41(4):725-9; discussion 725-9.

5. Pierro A, Hall $N$ : Surgical treatments of infants with necrotizing enterocolitis. Semin Neonatol 2003; 8 (3): 223-32.

6. Hall $N$, Eaton $S$, Pierro A: The evidence base for neonatal surgery. Early Hum Dev 2009; 85: 713-9.

7. Pierro A: The surgical management of necrotising enterocolitis. Early Hum Dev 2005; 81 (1): 79-85.

8. Cheng W, Leung MP, Tam PK: Surgical intervention in necrotizing enterocolitis in neonates with symptomatic congenital heart disease. Pediatr Surg Int 1999; 15 (7): 492-5.

9. O’Connor A, Sawin RS: High morbidity of enterostomy and its closure in premature infants with necrotizing enterocolitis. Arch Surg 1998; 133 (8): 875-80.

10. Harberg FJ, McGill CW, Saleem MM, Halbert R, Anastassiou $P$ : Resection with primary anastomosis for necrotizing enterocolitis. J Pediatr Surg 1983; 18 (6): 743-6.

11. Kiesewetter WB, Taghizadeh F, Bower RJ: Necrotizing enterocolitis: is there a place for resection and primary anastomosis? J Pediatr Surg 1979;14: 360-2. 